\title{
Cerebral metastasis during remission after immunotherapy for metastatic urothelial carcinoma: A case report
}

\author{
SHOHEI IWATA, FUSAKO NIIMI, TETSUYA DANNO, SACHI HONDA and TAKESHI AZUMA \\ Department of Urology, Tokyo Metropolitan Tama Medical Center, Fuchu, Tokyo 183-0042, Japan
}

Received January 16, 2021; Accepted April 1, 2021

DOI: $10.3892 / \operatorname{mco} .2021 .2307$

\begin{abstract}
Central nervous system (CNS) metastasis of urothelial carcinoma (UC) is rare. Immune checkpoint inhibitors, which were developed for the treatment of patients with advanced cancer, have limited efficacy against CNS metastases due to the unique immune microenvironment of the brain. The brain is an immune-privileged organ and is protected by the blood-brain barrier. However, the management of CNS metastases of UC is crucial to improving the prognosis. The present report describes two cases of cerebral metastasis occurring in the context of systemic disease control using immunotherapy. To the best of our knowledge, the present report is the first to describe a CNS metastasis during remission induced by immunotherapy.
\end{abstract}

\section{Introduction}

Central nervous system (CNS) metastasis of urothelial carcinoma (UC) is rare. In patients with metastatic UC receiving systemic chemotherapy, a cerebral metastasis sometimes occurs during a long follow-up period after chemotherapy, and the prognosis is usually very poor (1). Recent development of new types of systemic chemotherapy against UC, as well as improvements in immune checkpoint inhibitors for patients with advanced, metastatic forms of the disease, have contributed to improving the prognosis of patients. As immunotherapy can produce long-term remission in some patients, screening for CNS metastasis is important. However, to the best of our knowledge, the present report is the first to describe a CNS metastasis during remission induced by immunotherapy. We herein presented two cases of cerebral metastasis occurring in the context of systemic disease control by immunotherapy. To the best of our knowledge, the present report is the first

Correspondence to: Dr Takeshi Azuma, Department of Urology, Tokyo Metropolitan Tama Medical Center, 2-8-29 Musashidai, Fuchu, Tokyo 183-0042, Japan

E-mail: tazuma-tky@umin.ac.jp

Key words: immune checkpoint inhibitor, urothelial carcinoma, central nervous system metastasis, blood-brain barrier to describe a CNS metastasis during remission induced by immunotherapy.

\section{Case report}

Case 1. A 54-year-old, male patient with asymptomatic gross hematuria was referred to us by the previous hospital. He had no past history of any serious illness or smoking. Cystoscopy revealed a non-papillary, sessile bladder tumor, and computed tomography (CT) revealed invasive bladder cancer but no metastasis (cT2NOM0).

Transurethral resection of the bladder tumor (TURBT) was performed. Pathological examination showed Grade 3, pT2, UC. The patient was referred to our hospital for radical treatment. Because the patient declined a radical cystourethrectomy, intra-arterial chemotherapy with cisplatin and therarubicin for the bladder was performed in two cycles. After the intra-arterial chemotherapy, cystoscopy detected two recurrences of the bladder cancer over four months, prompting TURBT to be performed each time. One year after the intra-arterial chemotherapy, CT revealed a lung metastasis, and systemic chemotherapy with gemcitabine and cisplatin (GC) was begun. After four cycles, partial resection of the right middle lung lobe was performed. Pathological analysis revealed UC. By postoperative four months, the primary bladder cancer had grown, and new, multiple, lung metastases had appeared. Although an additional two cycles of GC chemotherapy were administered, the lung metastases and primary bladder cancer continued to grow (Fig. 1).

Immunotherapy with pembrolizumab was administered as the second line treatment. After six cycles, the primary bladder cancer and lung metastases resolved completely (Fig. 1), and immunotherapy was temporarily halted. Brain magnetic resonance (MRI) imaging at this time revealed no brain metastasis.

Two years after the immunotherapy with pembrolizmab, the patient was referred to our hospital due to aphasia. Brain MRI revealed a tumor in the left parietal lobe with edema (Fig. 2). Based on these findings, a cerebral metastasis was diagnosed, and irradiation therapy with Gamma Knife (27 Gy) was performed. The patient was recurrence-free 18 months after the radiation therapy.

Case 2. A 77-year-old, male patient with asymptomatic gross hematuria was referred to our hospital. He had a past history of diabetes mellitus and no history of smoking. Cystoscopy 
revealed a non-papillary, sessile bladder tumor on the left ureteral orifice. CT and MRI also revealed a left renal pelvic tumor with hydronephrosis and renal stone but no metastasis (Fig. 3). Based on these findings, bladder cancer and left renal pelvic cancer (cTaNOM0) were diagnosed preoperatively.

TURBT was performed, and a subsequent pathological examination revealed Grade 2, pTa, UC. Three months later, a left radical nephroureterectomy was performed, and pathological analysis revealed Grade 3, pT1, UC. Thereafter, TURBT was repeated three times over a year, and the pathological findings were Grade 3, pT1, each time. Because the patient declined a radical cystourethrectomy, three cycles of intra-arterial chemotherapy with cisplatin and therarubicin for the bladder cancer were performed. Subsequently, two more recurrences were detected in four months, and TURBT was performed on each occasion. The pathological analysis findings demonstrated Grade 3, pT1. One month later after the last TURBT, a radical cystourethrectomy with ileal conduit urinary diversion was performed.

Six years after the cystectomy, CT demonstrated a right lung tumor (Fig. 4). A bronchoscopic biopsy was performed, with the pathological findings revealing metastatic urothelial carcinoma. Positron emission tomography (PET) also showed abnormal uptake in the right lung tumor and mediastinal lymph nodes. Chemotherapy with gemcitabine and carboplatin was begun. However, the patient was unable to continue the treatment due to fatigue. As the second line treatment, immunotherapy with pembrolizumab was administered. At this time, brain MRI revealed no brain metastasis. After nine courses of immunotherapy, all the metastases had resolved, and immunotherapy was temporarily halted (Fig. 4).

Fifteen months after the termination of pembrolizumab treatment, the patient was referred to our hospital due to motor difficulties and disorientation. Neurological examination revealed no apparent focal deficits. Brain CT revealed multiple, low-density areas in the left frontal, parietal, and temporal lobes with edema (Fig. 5). Based on these findings, multiple, cerebral metastases were diagnosed, and whole brain irradiation therapy (30 Gy) was performed. Thereafter, the patient's disorientation improved.

\section{Discussion}

UC metastases commonly occur in the lungs, liver, lymph nodes, bone, adrenal glands, spleen, and intestine. CNS metastases are rare, with an incidence of less than $1 \%$ (1). However, the rate of CNS metastasis has meanwhile apparently increased to $16 \%$ (1). Platinum-based regimens have recently become the standard treatment for metastatic UC and have improved the prognosis of patients with this disease, but while chemotherapy can help achieve systemic remission, chemotherapeutic agents cannot cross the blood-brain barrier (BBB) to treat metastases in the brain (2), thus leading to the apparent increase in the incidence of CNS metastases of UC. In the present cases, CNS metastases were discovered 15-20 months after CR induced by immunotherapy, lending support to the above hypothesis.

Immune checkpoint inhibitors were recently developed for the treatment of patients with advanced cancer. With this new therapeutic modality, patients can achieve CR, after which immune surveillance can be deployed to insure long-term

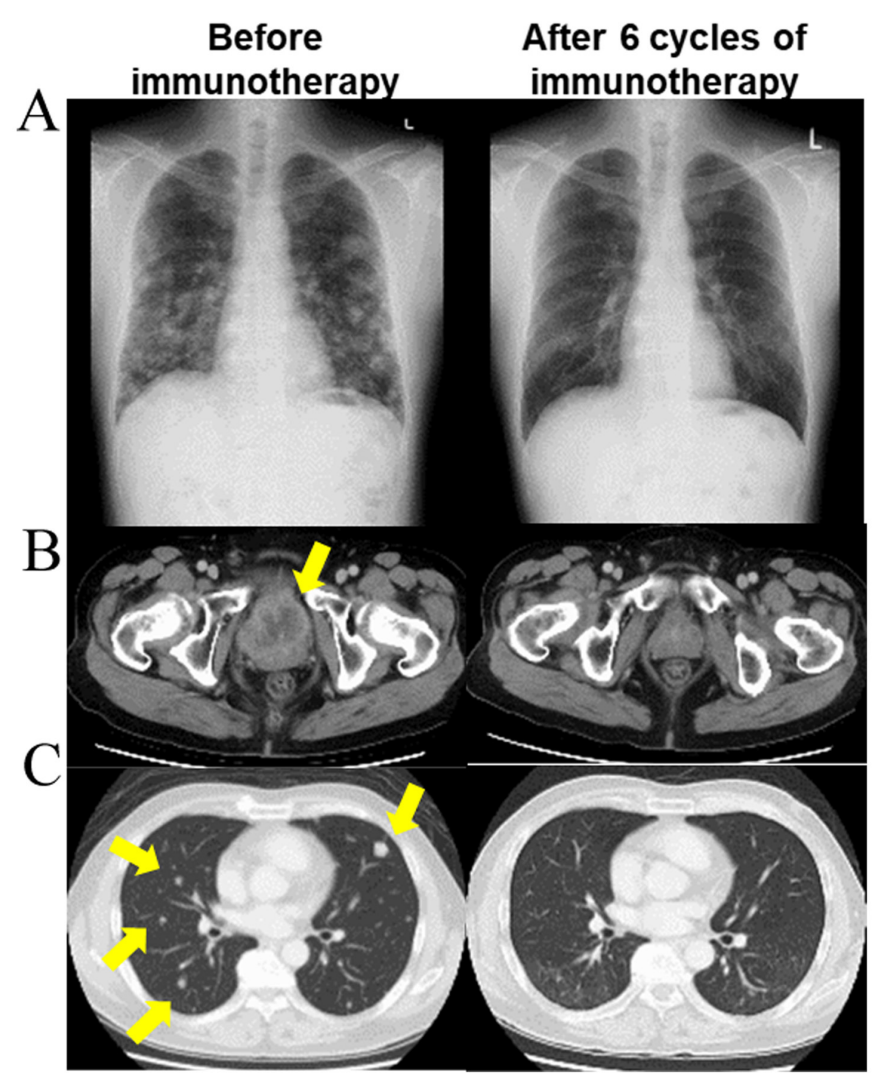

Figure 1. CXR and CT findings in case 1. These demonstrated resolution of the primary bladder tumor (arrow) (B) on CT and multiple lung metastases (arrows) (A) on CXR and (C) CT after six cycles of immunotherapy. CT, computed tomography; CXR, chest X ray.

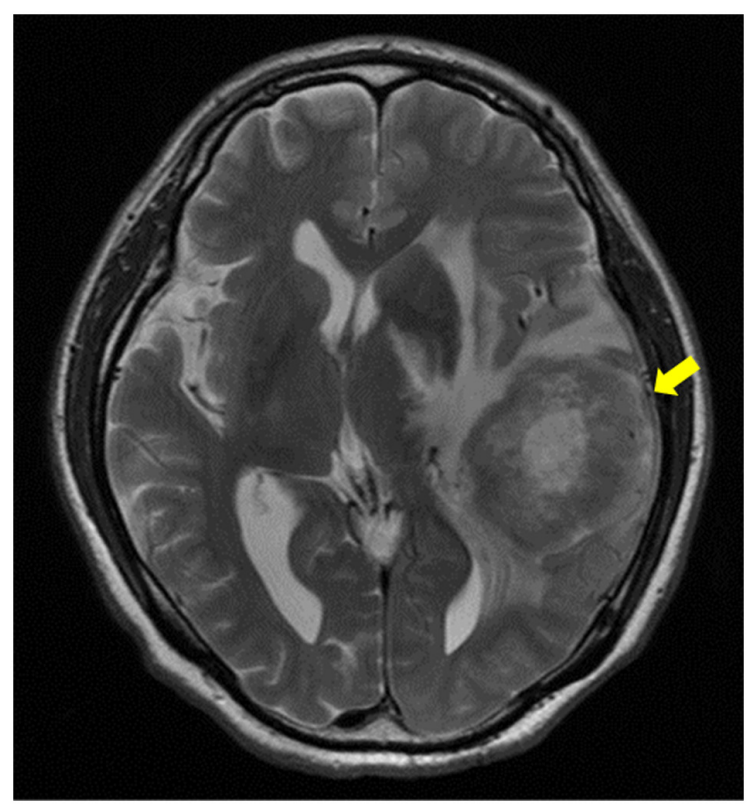

Figure 2. Brain T2-weighted MRI findings in case 1. Brain MRI revealed tumors (arrow) in the left parietal lobe with edema.

remission (3). However, immune checkpoint inhibitors have limited efficacy against CNS metastases thanks to the unique immune microenvironment of the brain (4). The blood-brain barrier prevents cytotoxic T lymphocytes (CTLs) from infil- 


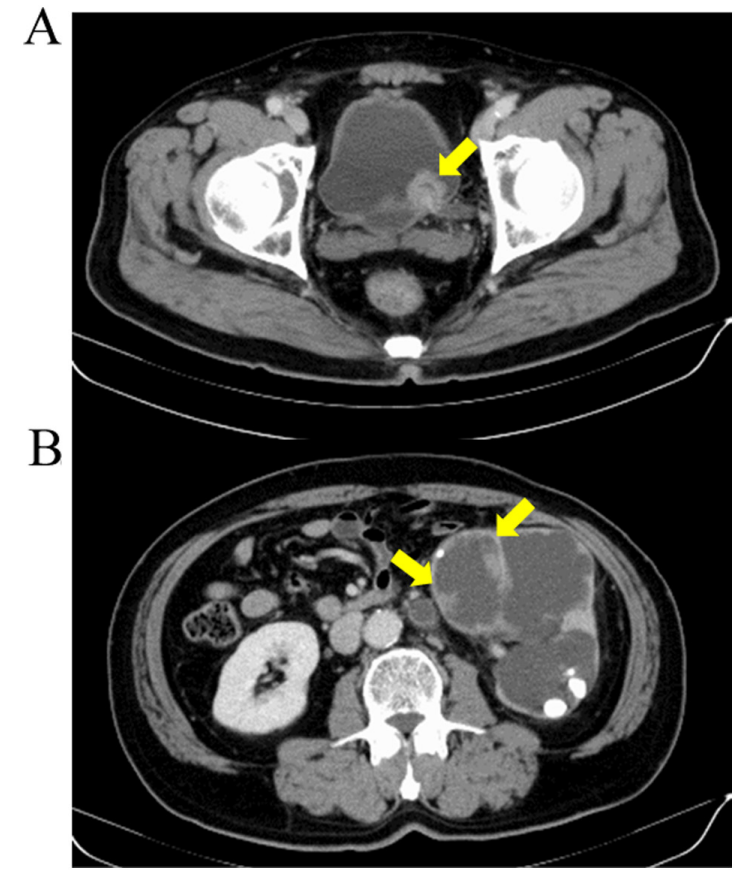

Figure 3. CT findings in case 2. CT demonstrated (A) a bladder tumor (arrow) on the left ureteral orifice and (B) a pelvic tumor (arrows) with hydronephrosis and renal stone. CT, computed tomography.

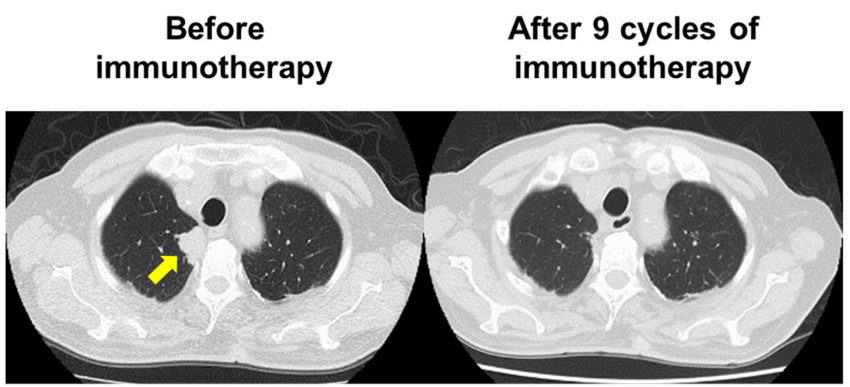

Figure 4. CT findings in case 2 showing new lung lesions (arrow). CT demonstrated resolution of the lung metastasis after nine cycles of immunotherapy. $\mathrm{CT}$, computed tomography.

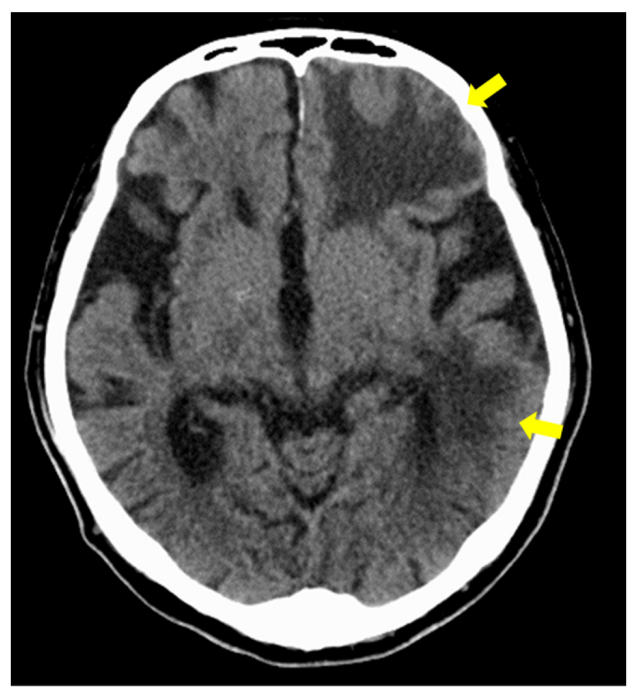

Figure 5. Computed tomography findings in case 2 demonstrating cerebral metastasis (arrows). The brain MRI revealed multiple, low density areas in the left frontal and temporal lobes with edema. trating brain metastases across the BBB. Even if long-term remission is achieved via immune checkpoint inhibitors, CNS metastases can elude treatment and continue growing owing to the brain's unique immune microenvironment. The following two facts in both of the present cases corroborate this hypothesis. First, the cerebral metastases developed more than one year after CR by immunotherapy. Second, no metastases were present in any of the other organs.

CNS metastases are detected with high regularity detected at our hospital. In two of three patients (66\%) in whom a cerebral metastasis of UC developed, complete, as well as long-term, remission was achieved via immunotherapy. In both patients, neurological symptoms prompted a CNS evaluation, suggesting that periodic evaluations of CNS metastasis status even in the absence of symptoms is more important for long-term disease control.

Radiotherapy was performed for the CNS metastasis in both of the present cases. It is likely that the radiotherapy was not only cytotoxic to the tumor cells, but also compromised the integrity of the BBB, possibly enabling CTLs to infiltrate the tumors and attack the cancer cells. Moreover, the subsequent immune surveillance reduced the risk of recurrence of CNS metastases. A previous study demonstrated that the prognosis of patients with brain recurrence was very poor, and that the median survival time usually did not exceed three months (1). In contrast, one of our patients was able to maintain remission for more than 12 months.

The present report has some limitations. First, the timing of the occurrence and the time course of the CNS metastases were unclear in both cases. Second, an appropriate screening schedule for CNS metastases was lacking. Third, the follow-up period was not long enough to determine whether there were any secondary effects due to the radiotherapy.

Although CNS metastases are very rare in patients with metastatic UC, evaluating for brain metastases is necessary, given its important role in disease control, especially in patients who have achieved CR and long-term remission by immunotherapy.

\section{Acknowledgements}

Not applicable.

\section{Funding}

No funding was received.

\section{Availability of data and materials}

The datasets used and/or analyzed during the current study are available from the corresponding author on reasonable request.

\section{Authors' contributions}

TA, SI and SH made substantial contributions in conception, design and interpretation of data. TA and SI wrote the manuscript. TD, FN and SH made substantial contributions in interpretation and acquisition of data. TA and SI were responsible for confirming the authenticity. All authors read and approved the final manuscript. 


\section{Ethics approval and consent to participate}

Not applicable.

\section{Patient consent for publication}

Both patients provided written informed consent for the publication of their data.

\section{Competing interests}

The authors declare that they have no competing interests.

\section{References}

1. Dhote R, Beuzeboc P, Thiounn N, Flam T, Zerbib M, Christoforov B and Debré B: High incidence of brain metastases in patients treated with an M-VAC regimen for advanced bladder cancer. Eur Urol 33: 392-395, 1998.
2. Anderson RS, el-Mahdi AM, Kuban DA and Higgins EM: Brain metastases from transitional cell carcinoma of urinary bladder. Urology 39: 17-20, 1992.

3. Swann JB and Smyth MJ: Immune surveillance of tumors. J Clin Invest 117: 1137-1146, 2007.

4. Ratnam NM, Gilbert MR and Giles AJ: Immunotherapy in CNS cancers: The role of immune cell trafficking. Neuro Oncol 21: 37-46, 2019. 